\title{
The prevalence of polycystic ovarian syndrome in Chinese women: a meta-analysis
}

\author{
Qiao Wu, Jing Gao, Dingxi Bai, Zhi Yang, Qing Liao \\ School of Nursing, Chengdu University of Traditional Chinese Medicine, Chengdu, China \\ Contributions: (I) Study conception and design: All authors; (II) Administrative support: J Gao; (III) Provision of study materials or patients: Q Wu, \\ Z Yang; (IV) Collection and assembly of data: Q Wu, Z Yang, Q Liao; (V) Data analysis and interpretation: Q Wu, D Bai, Z Yang; (VI) Manuscript \\ writing: All authors; (VII) Final approval of the manuscript: All authors. \\ Correspondence to: Jing Gao. School of Nursing, Chengdu University of Traditional Chinese Medicine, 1166, Liutai Avenue, Wenjiang, Chengdu \\ 611137, China. Email: 729012934@qq.com.
}

Background: To estimate the prevalence of polycystic ovarian syndrome (PCOS) in Chinese women comprehensively and to provide references to prevent PCOS.

Methods: China National Knowledge Infrastructure, Wanfang Data, China Science and Technology Journal Database, Chinese Biology Medicine, PubMed, Embase, Web of Science, CENTRAL, CINAHL were searched to collect studies on the prevalence of PCOS from database inception from March 31, 2020. Two reviewers independently screened literature according to the inclusion and exclusion criteria of the articles, extracted data, and tested the risk of bias of the included studies. Then Stata 15.1 software performed a meta-analysis. Begg's and Egger's method were used to assess the risk of publication bias.

Results: A total of 69 studies were involved, including 154,599 participants, 12,845 patients developed PCOS. The prevalence of PCOS was $10.01 \%$ (95\% CI: $8.31 \%$ to $11.89 \%$ ). Subgroup analysis showed that the prevalence of PCOS in different regions was as follows: $13.35 \%$ in the west, $7.82 \%$ in the east, $14.24 \%$ in the middle, and $8.68 \%$ in the north-east. Subgroup analysis by occupation/status: the prevalence rates of workers, students, medical staff, cadres, farmers, staff, and service staff were respectively $10.37 \%$, $16.37 \%, 13.97 \%, 6.05 \%, 7.05 \%, 8.05 \%, 9.05 \%$. For individuals aged 10 to 20, between 21 and 30,31 to 40 , and above 40 , the prevalence rate was $10.26 \%, 17.23 \%, 9.13 \%, 2.22 \%$, respectively. The prevalence rates of PCOS 1995 to 2000 were $21.04 \%$, from 2001 to 2005 , was $5.86 \%$, from 2006 to 2010 was $10.78 \%$, from 2011 to 2015 was $11.44 \%$, and during 2016 to 2020 was $5.79 \%$. Hospital-based community-based and school-based studies were $13.41 \%, 5.95 \%, 3.01 \%$. According to the subgroup analysis of the surveyed population, the prevalence of PCOS in the general population and infertility patients were $6.05 \%$ and $13.69 \%$, respectively

Conclusions: The prevalence rate of PCOS is high in Chinese women, and the prevalence of PCOS varies with different regions, occupation/identity, age, time of publication, diagnostic criteria, survey time, and prevalence of the surveyed population.

Keywords: Chinese woman; polycystic ovarian syndrome (PCOS); prevalence; meta-analysis

Submitted Aug 25, 2020. Accepted for publication Dec 29, 2020.

doi: 10.21037/apm-20-1893

View this article at: http://dx.doi.org/10.21037/apm-20-1893 


\section{Introduction}

Polycystic ovarian syndrome (PCOS) is the most common gynecological, endocrine system disease among women aged 18 to 44 (1). Its key characteristics are persistent anovulation, hyperandrogenism, and insulin resistance. The main manifestations are menstrual disorders and reproductive dysfunction (2-4), one of the leading causes of females in anovulatory infertility $(5,6)$. The World Health Organization estimates PCOS has affected over 116 million women worldwide as of 2010 (7). The incidence of longterm complications, including cardio-cerebrovascular diseases, hypertension, lipid metabolism disorders, and endometrial cancer in patients with PCOS, is 2 to 6 times ordinary people (2). At present, the etiology of PCOS is still unclear. Most scholars believe it may be related to the abnormal regulation function of the hypothalamicpituitary-ovarian axis, hyperinsulinemia and insulin resistance, abnormal adrenal endocrine function, heredity, and other aspects $(8,9)$. For decades, because of the differences in diagnostic criteria, sampling methods, and the gradual maturity of diagnosis and treatment techniques, the prevalence rates were retrieved by domestic and foreign scholars in the epidemiological investigations on PCOS vary greatly. Previous studies have reported that the prevalence rate of PCOS among women of childbearing age in various countries is between $5 \%$ and $10 \%(10)$. In recent years, large-scale investigations of PCOS have been carried out in different regions of China (11-13). However, due to the lack of a comprehensive data collection and analysis system, there is still no data on the exact frequency, distribution pattern and trend of PCOS nationwide. This study used the research method of meta-analysis to review the latest evidence systematically from the regional population survey of PCOS to understand the prevalence of PCOS in different regions and different populations fully, thus contributing to its control and management. We present the following article in accordance with the PRISMA reporting checklist (available at http://dx.doi.org/10.21037/apm-20-1893).

\section{Methods}

\section{Search strategy}

This study will be conducted according to PRISMA guidelines. Eight databases including China National Knowledge Infrastructure, Wanfang Data, China Science and Technology Journal Database, Chinese Biology Medicine, PubMed, Embase, The Cochrane Library and
Web of Science were searched to collect studies on the prevalence of PCOS in Chinese women from setting up a database to May 31, 2020. To increase the sensitivity, we use a combination of subject heading terms and free text words to retrieve documents. The subject headings include: "polycystic ovarian syndrome" and "prevalence" or "incidence" or "epidemiology" and "China" or "Chinese." The free words include: "The People's Republic of China" or "Mainland China" "Formosa" and "Stein-Leventhal Syndrome" or "Sclerocystic Ovarian Degeneration" or "Sclerocystic Ovary" and "occurrence" or "outbreaks" or "prevalence" or "endemics" or "incidence".

\section{Inclusion and exclusion criteria}

The inclusion criteria are: (I) type of study: prospective or retrospective studies on the prevalence of PCOS in Chinese women published at home and abroad; (II) study population: Chinese women with a definite diagnosis of PCOS; (III) outcome indicators and the prevalence of PCOS.

The criteria for exclusion were: (I) studies with incomplete information and data that cannot be extracted; (II) studies reported or published repeatedly in the same research population, excluding those of lower quality; (III) studies not in Chinese or English; (IV) study where only the abstract is visible, and the full text cannot be obtained by contacting the author; (V) studies with low quality (the AHRQ score is below 3 ).

\section{Data extraction}

Two investigators (Q Wu, Z Yang) independently screened the literature, extracted data, and cross-checked. If the two researchers have inconsistent conclusions, then through discussion to resolve differences. If the differences cannot be resolved, the third party will perform judgments and arbitration. First, read the title and abstract, and after excluding irrelevant studies, read the full text further to determine whether it will be included. The content of the data extraction includes the first author, publication year, study design, research area, age, number of PCOS cases and sample size, occupation, education background, diagnostic criteria.

\section{Statistical analysis}

STATA (version 15.1; Stata Corporation) software was used to conduct statistical analysis and the point 


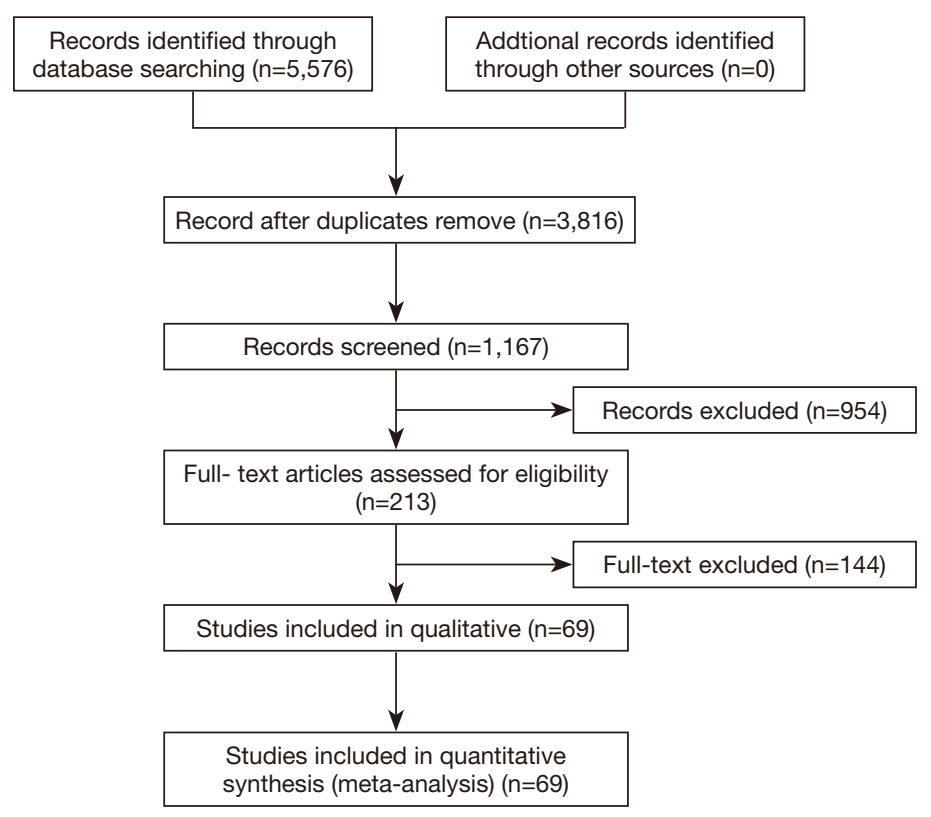

Figure 1 The flow diagram of this meta-analysis.

prevalence rates were first transformed into arcsine squareroot transformed proportions (14). The heterogeneity between the included studies was determined by the $\mathrm{Q}$ test ( $\mathrm{P}$ value) and $\mathrm{I}^{2}$. If $\mathrm{I}^{2}<50 \%$ and $\mathrm{P}>0.1$ of the $\mathrm{Q}$ test, it is considered the homogeneity between the studies is satisfactory, the fixed-effect model is used for meta-analysis. On the contrary $\left(\mathrm{P} \leq 0.1\right.$ or $\left.\mathrm{I}^{2} \geq 50 \%\right)$, it is considered there is evident heterogeneity among studies, and the random effects model is used for combined calculation. A subgroup analysis was performed to explore the possible sources of heterogeneity among studies. Also, the subgroup analysis was conducted according to different regions, occupation/ identity, age, publication time, diagnostic criteria, survey time, and survey population. Begg's test and Egger's test were used for conducting a publication bias assessment. It is considered statistically significant when $\mathrm{P}<0.05$. In addition, a sensitivity analysis was performed to measure the impact of any research on aggregate estimates.

\section{Quality assessment}

The quality of the included studies was tested using the cross-sectional study quality evaluation standards recommended by the Agency for Healthcare Research and Quality (AHRQ). The AHRQ (15) standard contains 11 items, which are answered with "yes", "no," and "do not know", respectively. If the answer is "yes", the item is judged as "1", "no" or "no." "Clear" is " 0 " points. The score ranges from 0 to 11 points, 0 to 3 are low quality, 4 to 7 are medium quality, and 8 to 11 are of high quality. The literature quality evaluation was conducted independently by two researchers (Qiao $\mathrm{Wu}$ and Qin Liao), and the results were cross-checked. If the conclusions of the two researchers were inconsistent, a consensus was reached through discussion.

\section{Results}

\section{Study selection and participants characteristics}

The literature screening process and results are shown in Figure 1. A total of 5,576 papers were obtained from the preliminary search, among which 265 were from PubMed database, 108 from Embase, 708 from Web of Science, CENTRAL 33, CNKI 1,071, WanFang Data 1,479, VIP 667 and CBM 1,345. After reading the title, abstract and eliminating duplicate studies, a total of 4,409 articles were excluded. Then after further reading the full text, according to the literature inclusion and exclusion criteria, 62 documents whose data could not be extracted were excluded, 15 documents whose document type was review, 29 articles with a quality score of less than 3 , and 21 documents with duplicate data, 17 documents without a clear survey location, 69 articles were finally included papers 
on PCOS prevalence in Chinese women (65 in Chinese and 4 in English). A total of 154,599 patients were enrolled, including 12,845 patients with PCOS. The literature includes 23 provinces (cities, autonomous regions, and particular administrative regions), including 15 Midwestern Sectional Figure Skating Championships, 33 in the Eastern Region, 16 in the central region, and 4 in the northeast. Among them, three were of low quality, and 57 were of medium quality, and five were of high quality. The primary characteristics of the included studies are shown in Table 1.

\section{Pooled prevalence rates of PCOS}

Sixty-nine articles, including 154,112 subjects and 12,845 patients, were included. The prevalence rate of PCOS reported in each study ranged from $0.45 \%$ to $35.14 \%$. A heterogeneity test was performed on the included 69 articles, and there was significant heterogeneity among the articles $\left(\mathrm{I}^{2}=99.6 \%, \mathrm{P}<0.05\right)$, so the random effects model was adopted. From the fact that there are more ratios in this study between $0-0.3$ and $0.7-1$, the double arcsine conversion method was used to convert the rates and then combine to calculate the prevalence. The combined conversion rate from the random effects model was 0.64 (95\% CI: $0.58-0.70$ ), and the total prevalence of PCOS in Chinese women was $10.01 \%$ (95\% CI: $8.31-11.89 \%$ ) (Figure 2).

\section{Subgroup analysis}

Subgroup analysis was conducted by region, occupation/ identity, age, time of publication, diagnostic criteria, and survey population. The study area is divided into eastern, northeastern, central, and western regions, according to China's four economic zones. Occupations/identities are divided into workers, students, medical personnel, cadres, farmers, clerks, and service personnel. The age is divided into 10-20, 21-30, 31-40 years old, and over 40 years old. Research sites are divided into hospitals, communities, and schools. The year of publication is in a period of every five years. Subgroup analysis results are shown in Table 2.

\section{Sensitivity analysis and publication bias}

Sensitivity analysis showed there was no significant difference between the results obtained and the total combined estimate when each of the included articles was excluded one by one, showing that the results were stable.
Funnel chart and Begg's method and Egger funnel figure asymmetric inspections method analysis were performed. The results show Begg's rank correlation test income $=0.81$, $\mathrm{P}=0.41$, and $\mathrm{Z}$ suggests no publication bias, and $\mathrm{P}=0.01$ Egger regression analysis income. Further, the funnel chart shows apparent asymmetry, suggesting a potential publication bias, language bias, the small-scale flawed method in the study designed to estimate highly. Therefore, the effect of publication bias on the results was tested by the shear compensation method. The results showed that the estimated value of the combined effect size did not change significantly, suggesting that publication bias had little impact on the results, and the meta-analysis results were relatively stable (Figures 3,4).

\section{Discussion}

PCOS is the most common endocrine disease in women of childbearing age, and its clinical manifestations are highly heterogeneous (82), which can be easily confused with endocrine diseases, including hyperprolactinemia, abnormal thyroid function, and congenital adrenal hyperplasia (83). So far, there is no cure for PCOS, but the related health risks are lifelong. Even after menopause, women with PCOS still often have higher levels of androgen and insulin resistance $(3,9)$. In recent years, the incidence of PCOS has all increased (84), and with the improvement of diagnostic methods and the level of diagnosis and treatment, its prevalence may change accordingly. Therefore, this study intends to assess the prevalence of PCOS in Chinese adult women and analyze the time tend to determine the current prevalence of PCOS in Chinese women to decide for the prevention and treatment of PCOS to provide evidence.

Sixty-nine articles published from 1985 to 2019 were included in this study and described the prevalence of PCOS among Chinese women through the method of meta-analysis. The results show the total prevalence of PCOS in China is $10.01 \%$, which is higher than the results obtained by Rong $\mathrm{Li}$ (11) and others in the epidemiological survey of female PCOS in 10 provinces (cities). However, the prevalence of PCOS in this study was lower than that in Middle Eastern women (16\%) (85), which may be related to race, region, economic and cultural level, and the improvement of PCOS knowledge and diagnosis.

The study also found significant differences among regions, occupation/identity, age, time of publication, diagnostic criteria, and prevalence rates among survey populations. In terms of regional differences in the 
Table 1 The basic characteristics of the included studies

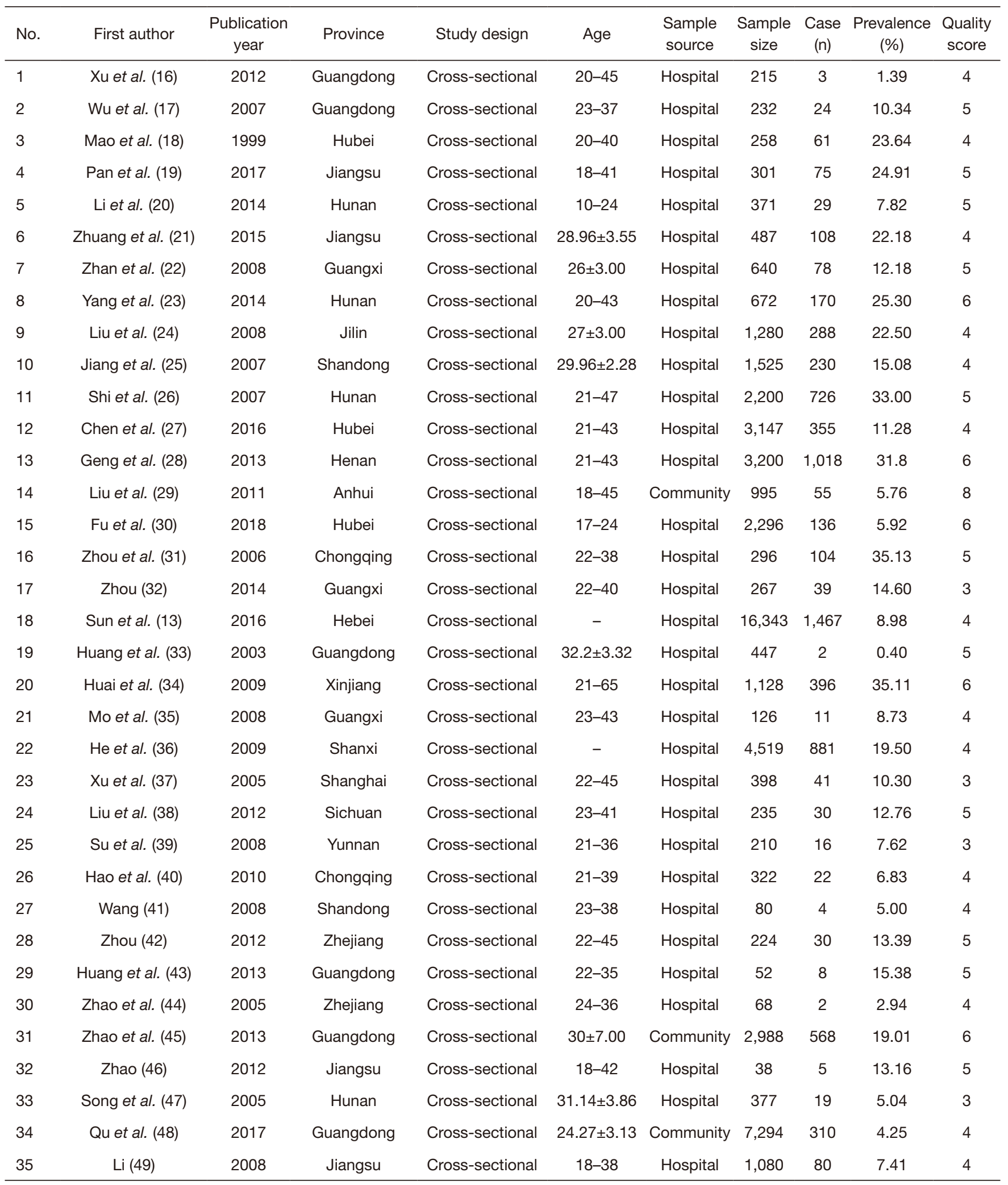

Table 1 (continued) 
Table 1 (continued)

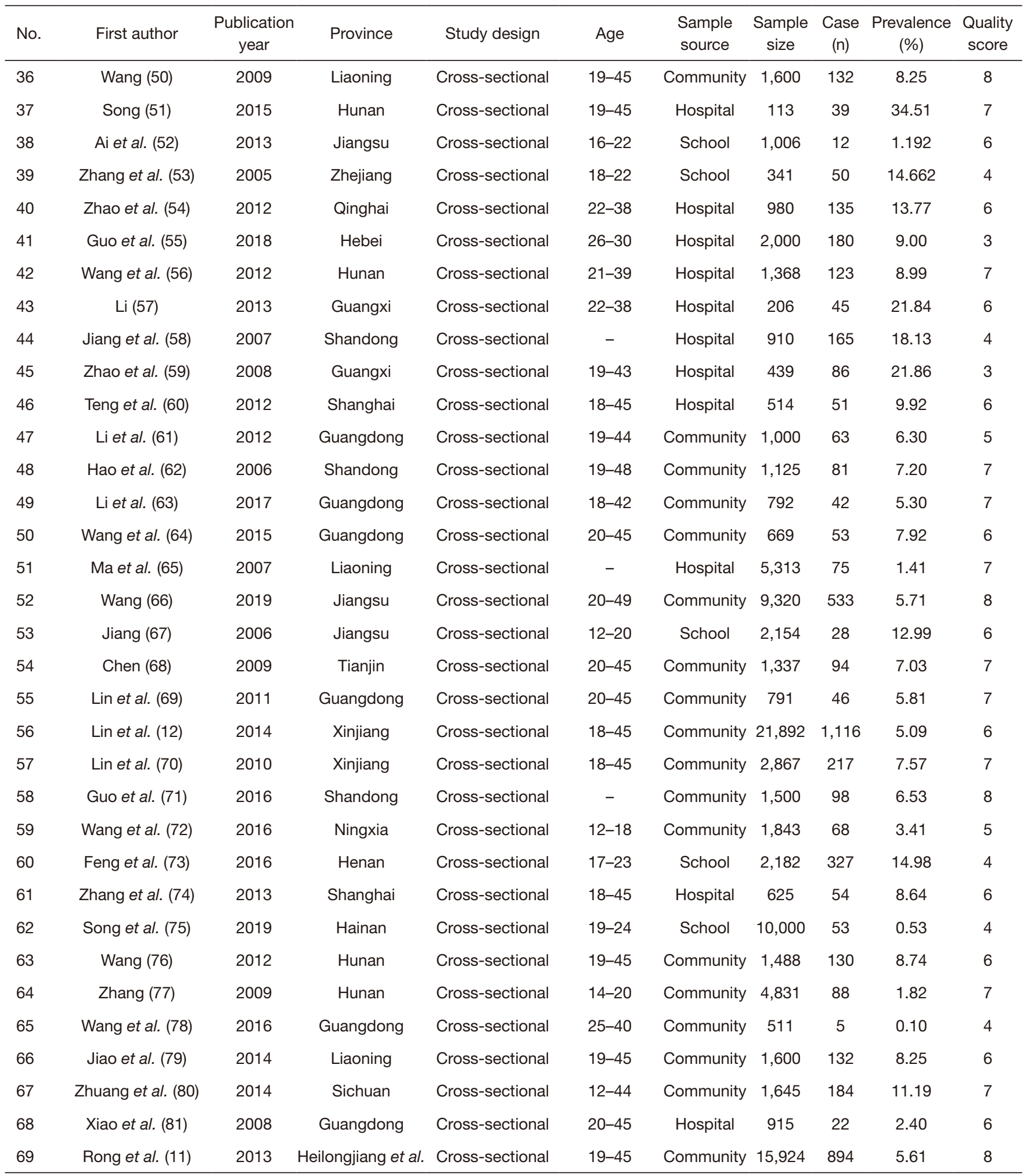

"-" not reported. 


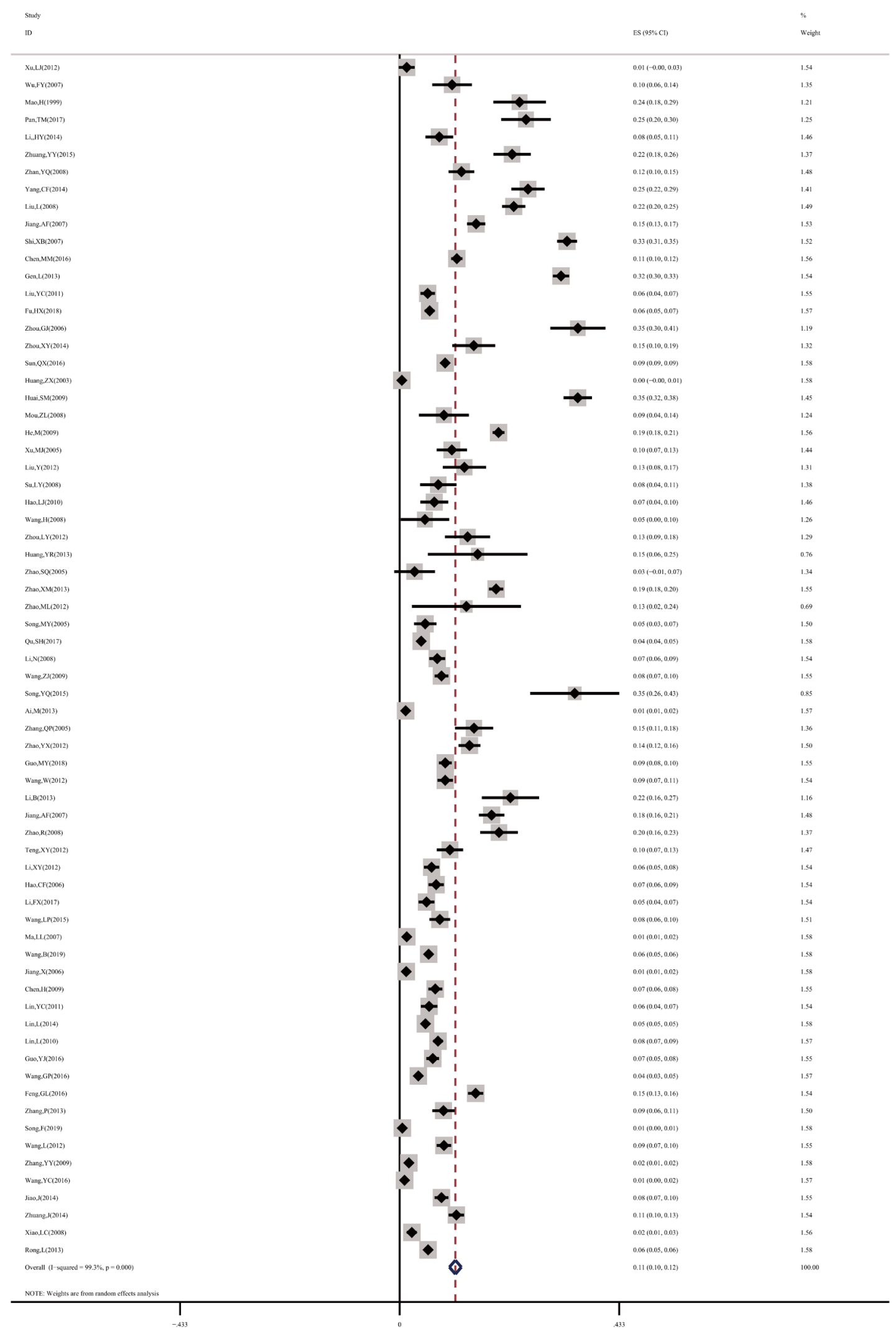

Figure 2 Forest plots of the total prevalence of polycystic ovary syndrome in Chinese women. 
Table 2 Subgroup analysis of the prevalence of polycystic ovary syndrome in Chinese women

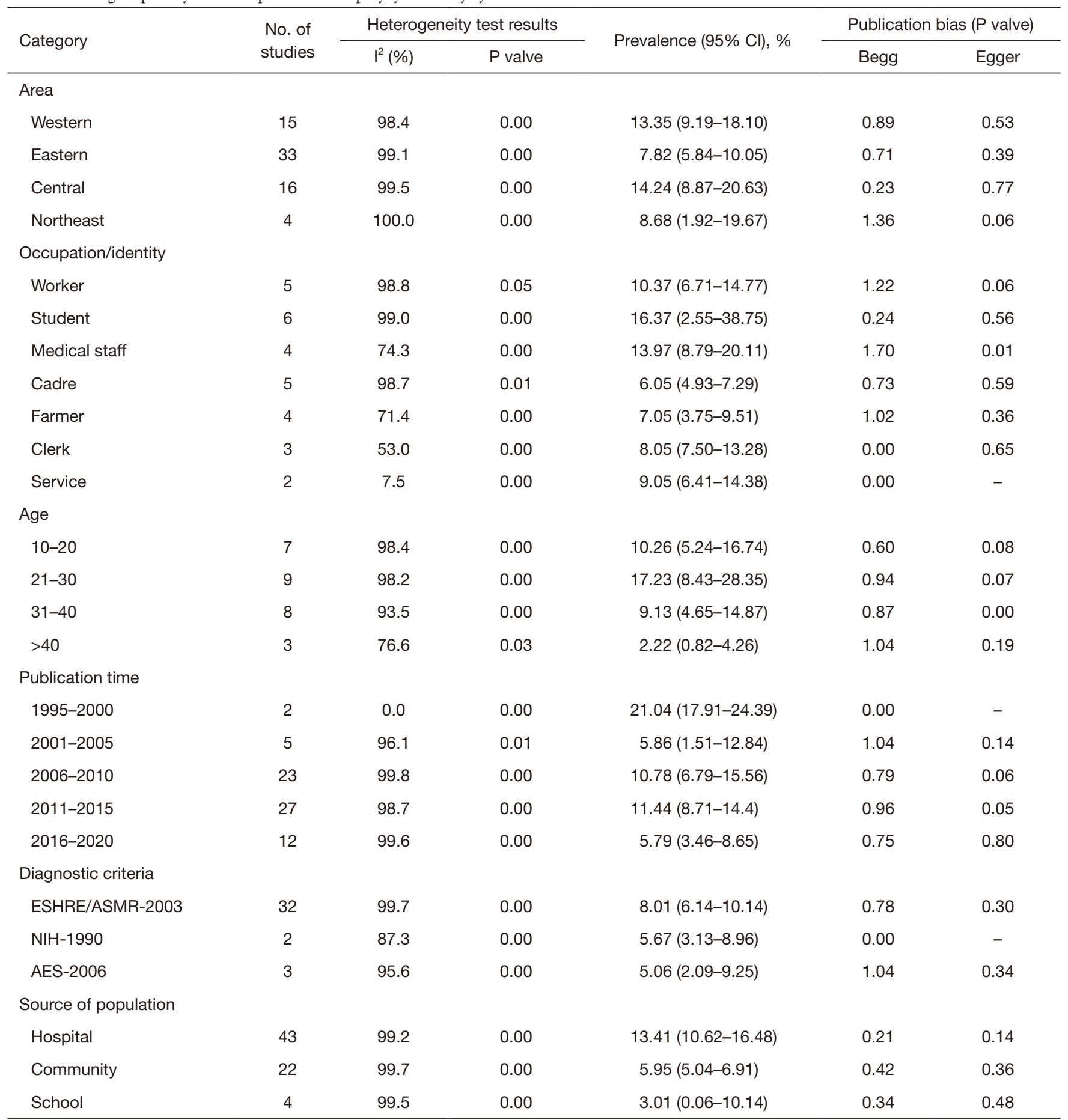

NIH-1990 (Diagnostic criteria developed by the United States Health Organization/United States Organization for Child Health and Human Development (NIH/NICHD) in 1990): (I) oligoovulation, (II) signs of androgen excess (clinical or biochemical), (III) exclusion of other disorders that can cause menstrual irregularity and hyperandrogenism. ESHRE/ASMR-2003 [Diagnostic criteria developed by the European Society of Human Reproduction and Embryology (ESHRE) and the American Society for Reproductive Medicine (ASRM) in 2003]: (I) oligoovulation and/or anovulation, (II) excess androgen activity, (III) polycystic ovaries (by gynecologic ultrasound) AES2006 (Diagnostic criteria developed by the American Androgen Excess Society in 2006): (I) excess androgen activity, (II) oligoovulation/ anovulation and/or polycystic ovaries, (III) exclusion of other entities that would cause excess androgen activity. 


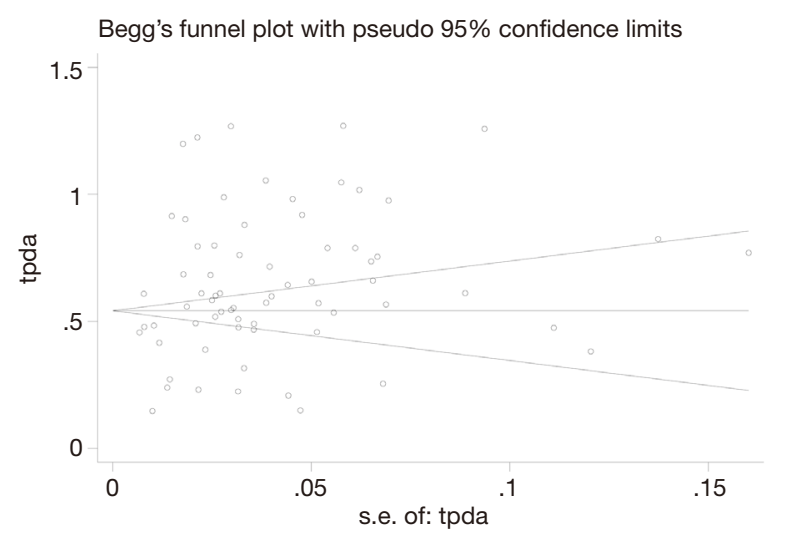

Figure 3 The funnel plot diagram of Beggar rank correlation analysis

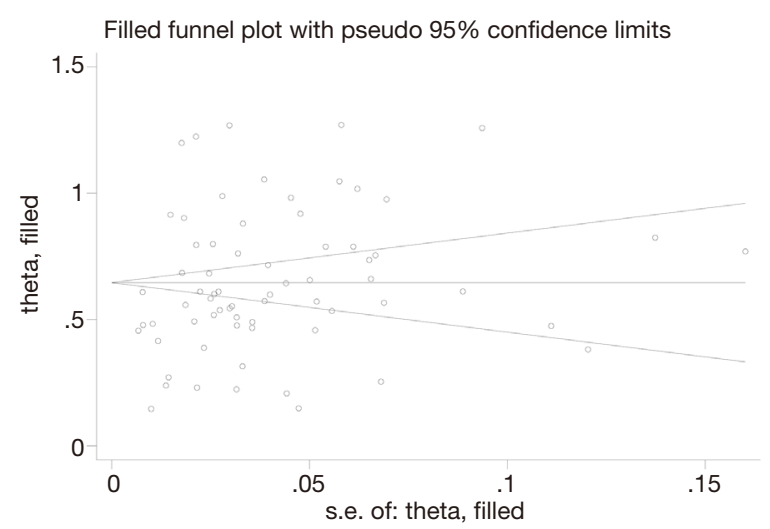

Figure 4 The funnel diagram of the shear compensation method.

prevalence rate of female PCOS, the prevalence rate of female PCOS in eastern regions is significantly lower than that in central and western regions, which might be because of the following reasons: (I) compared with the eastern region, the economic status of the central and western regions is slightly lagging. The socioeconomic status of women is low. Relevant studies have shown that low socioeconomic status is related to increased exposure to environmental BPA (86), and this synthetic compound may be related to PCOS (87). Relevant animal studies have shown (88) that there is an association between the development of PCOS symptoms and exposure to BPA during the newborn period. Simultaneously, women in the central and western regions are insufficient preventive and health care conditions because of the lagging economic status (89), which delays the treatment of PCOS. (II) It is related to the living environment between regions.
Women in the central and western regions are more likely to be in backward agricultural areas than women in the eastern regions and are more likely to be exposed to environmental toxins, including pesticides and smog. Studies have shown that environmental toxins have a significant impact on human health. Pollutants, including smoke, lead, pesticides, and mercury, may damage women's reproductive health and may trigger or aggravate PCOS and its related symptoms (90).

In terms of the difference in the prevalence of PCOS between different occupations/identities: PCOS has the highest prevalence among students. The reasons may be because of: (I) students have prolonged mental work hours and high intensity, and their bodies are under longterm stress. Catecholamine increased secretion causes the body's endocrine function disorder, sympathetic nerve excitability, and hypothalamus-pituitary-adrenal cortex hormone secretion increases, thereby reducing immune function. These factors may accelerate the occurrence of PCOS (91). (II) Students often have lousy living habits, including reversed work and rest and irregular eating and less exercise and exercise, and these are all predisposing factors for PCOS (92). As a group of people with higher socioeconomic status, cadres have better medical and health conditions and pay more attention to their health conditions, have a relatively healthier lifestyle, and reduce the chance of PCOS.

The results of a subgroup analysis with age in this study showed that the prevalence rate was $10.26 \%$ for 10 to 20 years old, $17.23 \%$ for women aged 21 to 30 , and $9.13 \%$ for women aged 31 to $40,>40$ years old. The prevalence of PCOS in women was $2.22 \%$. This result shows that the prevalence of PCOS decreased significantly with age from 21 to 30 years old, suggesting that age may be related to PCOS. The reason might be because of the prosperous childbearing period for women of childbearing age between 20 and 29 years old, female reproductive endocrine function reaches its peak state, the ovarian endocrine function is vigorous, and women's ovarian endocrine function enters a state of gradual decline around 40 years old (93). The prevalence of PCOS, which is closely related to endocrine function, also decreases with the gradual weakening of ovarian endocrine function.

In terms of publication time, from 2001 to 2015, the prevalence of female PCOS has shown an upward trend. The reason may be that with the development of economy and medical and health conditions, increased patients with PCOS-related symptoms tend to go to the hospital 
for treatment. Also, with the improvement of diagnosis and treatment methods, and the gradual improvement of PCOS diagnostic criteria, the diagnosis rate of PCOS has increased. The prevalence of PCOS has declined since 2016. The decline might be related to the changes in the age structure of the population in the country. The number of women of childbearing age in this study's country has shown a decreasing trend year by year. According to the 2018 Government Work Report, there were 4 million fewer women of childbearing age-aged 15 to 49 in 2017 than in 2016, with nearly 6 million fewer women of childbearing age-aged 20 to 29 during their fertile years.

Comparing the prevalence of women using the diagnostic criteria of the National Institutes of Health in 1990, the Rotterdam criteria in 2003 and the PCOS diagnostic criteria developed by the American Androgen Association in 2006, the prevalence of PCOS diagnosed using the Rotterdam criteria in 2003 is significant It is higher than the other two is like the results of researchers including Anahita Jalilian (10). However, some studies believe the Rotterdam criteria may include some women with mild phenotypes into the diagnosis, increasing the incidence of PCOS (94). In terms of the prevalence of PCOS in different survey sites, the survey conducted in the hospital has a higher prevalence rate, while the survey conducted in the school has a lower prevalence rate. The reason might be because of the differences in the subjects of the survey. Most surveys conducted in the hospital were women who had symptoms related to PCOS and went to the hospital for treatment. The cases were concentrated. The low prevalence of the survey conducted in schools may be related to insufficient self-health care, awareness of students, insufficient relevant knowledge of PCOS, and insufficient school medical and health conditions. When students have PCOS-related symptoms, including irregular menstruation and acne, they are often overlooked and miss the diagnosis and treatment of the disease.

This study also has certain limitations. Firstly, it is limited by the characteristics of single rate meta-analysis, and the heterogeneity between the literature is considerable. Secondly, significant heterogeneity exists among different studies. Although inclusion and exclusion criteria are strictly limited and subgroup analysis is conducted, the results cannot fully explain the source of heterogeneity, and unknown sources of heterogeneity may cause certain bias. Finally, only 69 studies covering 23 provinces in China were included in this study. The results were not enough to reflect the prevalence and characteristics of PCOS in all Chinese women.

\section{Conclusions}

To sum up, PCOS has a high prevalence rate in Chinese women, a significant public health problem in China. Therefore, medical and health departments should pay more attention to this situation. According to the research results, the disease has a high prevalence rate in the central region, students, and women aged 21 to 30 . Therefore, more targeted screening measures should be adopted for people with the characteristics of this population, and tertiary prevention of PCOS should be actively carried out, to make an early diagnosis and early treatment, and reduce relevant medical and health resources.

\section{Acknowledgments}

Funding: None.

\section{Footnote}

Reporting Checklist: The authors have completed the PRISMA reporting checklist. Available at http://dx.doi. org/10.21037/apm-20-1893

Conflicts of Interest: All authors have completed the ICMJE uniform disclosure form (available at http://dx.doi. org/10.21037/apm-20-1893). The authors have no conflicts of interest to declare.

Ethical Statement: The authors are accountable for all aspects of the work in ensuring that questions related to the accuracy or integrity of any part of the work are appropriately investigated and resolved.

Open Access Statement: This is an Open Access article distributed in accordance with the Creative Commons Attribution-NonCommercial-NoDerivs 4.0 International License (CC BY-NC-ND 4.0), which permits the noncommercial replication and distribution of the article with the strict proviso that no changes or edits are made and the original work is properly cited (including links to both the formal publication through the relevant DOI and the license). See: https://creativecommons.org/licenses/by-nc-nd/4.0/.

\section{References}

1. Nandi A, Chen Z, Patel R, et al. Polycystic ovary syndrome. Endocrinol Metab Clin North Am 
2014;43:123-47.

2. Kauffman RP, Baker TE, Baker VM, et al. Endocrine and metabolic differences among phenotypic expressions of polycystic ovary syndrome according to the 2003 Rotterdam consensus criteria. Am J Obstet Gynecol 2008;198:670.e1-7; discussion 670.e7-10.

3. Teede H, Deeks A, Moran L. Polycystic ovary syndrome: a complex condition with psychological, reproductive and metabolic manifestations that impacts on health across the lifespan. BMC Med 2010;8:41.

4. Ho CW, Chen HH, Hsieh MC, et al. Hashimoto's thyroiditis might increase polycystic ovary syndrome and associated comorbidities risks in Asia. Ann Transl Med 2020;8:684.

5. Azziz R, Woods KS, Reyna R, et al. The prevalence and features of the polycystic ovary syndrome in an unselected population. J Clin Endocrinol Metab 2004;89:2745-9.

6. Giviziez CR, Sanchez EG, Approbato MS, et al. Obesity and anovulatory infertility: A review. JBRA Assist Reprod 2016;20:240-5.

7. Vos T, Flaxman AD, Naghavi M, et al. Years lived with disability (YLDs) for 1160 sequelae of 289 diseases and injuries 1990-2010: a systematic analysis for the Global Burden of Disease Study 2010. Lancet 2012;380:2163-96.

8. De Leo V, Musacchio MC, Cappelli V, et al. Genetic, hormonal and metabolic aspects of PCOS: an update. Reprod Biol Endocrinol 2016;14:38.

9. Puurunen J, Piltonen T, Morin-Papunen L, et al. Unfavorable hormonal, metabolic, and inflammatory alterations persist after menopause in women with PCOS. J Clin Endocrinol Metab 2011;96:1827-34.

10. Jalilian A, Kiani F, Sayehmiri F, et al. Prevalence of polycystic ovary syndrome and its associated complications in Iranian women: A meta-analysis. Iran J Reprod Med 2015;13:591-604.

11. Li R, Zhang Q, Yang D, et al. Prevalence of polycystic ovary syndrome in women in China: a large communitybased study. Hum Reprod 2013;28:2562-9.

12. Lin L, Yang J, Ding Y. Prevalence of polycystic ovary syndrome of Uygur and Han population in Xinjiang of China. Journal of Hainan Medical College 2014;20:680-3.

13. Sun Q, Sui L, Xu Y. Adverse pregnancy outcome analysis of 507 cases with polycystic ovary syndrome. Chinese Journal of Primary Medicine 2016;23:298-300.

14. DerSimonian R, Laird NJ. Meta-analysis in clinical trials Control Clin Trials 1986;7:177-88.

15. Rostom A, Dubé C, Cranney A, et al. Celiac disease. Evid Rep Technol Assess (Summ) 2004;(104):1-6.
16. Xu L, Li L, Zheng X, et al. Clinical Analysis of Laparoscopy Diagnosis and Treatment in 215 Cases of Pelvic Diseases for Female Infertility. Journal Of Modern Clinical Medicine 2012;38:105-7.

17. Wu F, Huang D. Analysis of 232 cases of female infertility treated by laparoscopy. Journal of Guangdong Medical College 2007;25:310-1.

18. Mao H. Analysis of 258 cases of infertility diagnosed by laparoscopy. Acta Academiae Medicinae Hubei 1999;20:3-5.

19. Pan T, Cao S. Analysis of infertility factors in 301 couples. Journal of Practical Gynecologic Endocrinology 2017;4:105-6.

20. Li H, Zhou Y, Wu Y. Analysis of 371 cases of adolescent gynecological diseases. Medical information 2014;27:138-9.

21. Zhuang Y, Xia F, Zhou W, et al. Analysis of Thyroid Function of 487 Infertile Women. Chinese Journal of Hemorheology 2015;25:74-7.

22. Zhan Y. The Etiological Factor Analysis of 640 Cases Barrenness. Heilongjiang Medicine 2008;32:292-3.

23. Yang C, Zuo Q. Etiology analysis of endocrine sterility in 672 female patients. Contemporary Medicine 2014;20:28-30.

24. Liu L, Li Y, Tai S. The Etiological Factor Analysis of 1280 Cases with Female Infertility. China Modern Doctor 2008;46:71-2.

25. Jiang A, Reng C, Li Y. Etiology analysis of 1525 cases of infertility. Journal of Practical Obstetrics and Gynecology 2007;23:705-6.

26. Shi X, Fang X. Etiology analysis of 2200 infertility patients. Medical Clinical Research 2007;24:1316-7.

27. Chen $M, \mathrm{Xu}$ W. Etiology analysis of infertility in 3147 patients with ART assisted pregnancy treatment. Journal of Reproductive Medicine 2016;25:360-3.

28. Geng L, Guo Y, Yang Y. Analysis of clinical etiology of 3200 cases of infertility. Journal of Henan Medical College for Staff and Workers 2013;25:141-3.

29. Liu Y, Wei Z, Xu Y, et al. Characteristics of polycystic ovary syndrome in women of reproductive age in Anhui province. Journal of Reproductive Medicine 2011;20:178-82.

30. Fu H, Zhu H, Kang M. Study on Different Criteria for Diagnosis Polycystic Ovary Syndrome Among Female College Students. Labeled Immunoassays and Clinical Medicine 2018;25:894-7.

31. Zhou G. Analysis of 296 cases of sterility with TV laparoscopic surgery. Chinese Journal Of Misdiagnostics 
2006;6:548.

32. Zhou $X$. The results of laparoscopy in 267 infertility patients were analyzed. Contemporary Medicine Forum 2014:178-9.

33. Huang Z, Liao S, Xu K, et al. Clinical Application of Laparoscopic Surgery in Gynecology. Medical Journal Wuhan University 2003;24:291-2.

34. Huai S. Investigation and analysis of changes of endocrine diseases in gynecological outpatient department. Xinjiang: Xinjiang Medical University; 2009.

35. Mo X. The value of laparoscopy in the diagnosis and treatment of female infertility. Acta Medicinae Sinica 2008;21:279-81.

36. He M, Gao B, Zhao W, et al. Clinical analysis of laparoscopy in 4519 cases with female infertility. Chinese Journal of Practical Gynecology and Obstetrics 2009;25:691-3.

37. Xu M, Hu N, Cui Y, et al. Analysis of 398 female patients with infertility under laparoscopy. Chinese Journal of Endoscopy 2005;11:155-7.

38. Liu Y, Fu J, Tang B. Clinical Study of Laparoscopic Diagnosis and Treatment in 235 Cases with Female Infertility. Journal of Modern Clinical Medicine 2012;38:202-4.

39. Su L, Wang M, Ma Y, et al. Clinical analysis of laparoscopy in diagnosis and treatment of infertility. Chinese Journal of Birth Health And Heredity 2008;16:103.

40. Hao L, Lin Y, Sun W, et al. Retrospective analysis of laparoscopic diagnosis and treatment in female infertility. Chinese Journal of Family Planning 2010;18:620-2.

41. Wang H. Analysis of 80 cases of female infertility treated by laparoscopy. Shandong Medical Journal 2008;48:73-4.

42. Zhou L. Analysis of 238 cases of female infertility treated by laparoscopy. Chinese Journal of Rural Medicine and Pharmacy 2012;19:19-20.

43. Huang Y, Dai $Z$. Application value of laparoscopy in diagnosis and treatment of female infertility. International Medicine and Health Guidance News 2013;19:2536-7.

44. Zhao S, Ding H. Clinical analysis of 68 cases of pelvic infertility treated by laparoscopy. Modern Journal of Integrated Traditional Chinese and Western Medicine 2005;14:289-90.

45. Zhao X, Ni R, Huang J, et al. Study on the facial and body terminal hair growth in women in Guangdong by using modified Ferriman-Gallwey scoring system. Zhonghua Fu Chan Ke Za Zhi 2013;48:427-31.

46. Zhao $M$. The value of combined application of uterine and laparoscopy in the treatment of infertility. China Health
Industry 2012;000:144.

47. Song M, Zhou C, Ma W. An Analysis of 377 Infertile Patients Examined by Combined Laparoscopy and Hysteroscopy. Practical Preventive Medicine 2005;12:401-2.

48. Qu S, Xu J, Huang X, et al. An epidemiological survey of infertility among couples of childbearing age at first marriage in Yangjiang district, Guangdong Province. Guangdong Medical Journal 2017;38:1586-8.

49. Li N. Diagnosis and treatment of 1080 cases of secondary amenorrhea. Journal of Qiqihar Medical College 2008;29:820-1.

50. Wang Z. An epidemiological survey of polycystic ovary syndrome in Han women of childbearing age in Liaoning Province. Shenyang: China Medical University; 2009.

51. Song Y. Investigation and analysis of changes of endocrine diseases in outpatient department. Mother and Child World 2015;000:145.

52. Ai M, Lu Y, Wang A. A survey of menstrual and adolescent polycystic ovary syndrome among female students in a college. Maternal \& Child Health Care of China 2013;28:3327-30.

53. Zhang Q, Yu Q, Shen C, et al. Analysis report and countermeasure of 341 cases of female college students with B-ultrasonography in abdomen. Chinese Journal of Modern Obstetrics and Gynecology 2005;2:478-9.

54. Zhao Y. Analysis of 980 cases of related factors and causes for female infertility. Modern Preventive Medicine 2012;39:3528-9.

55. Guo M, Jiang C, Zhang H. Study on the prevalence and clinical characteristics of polycystic ovary syndrome in infertile women. Journal of Practical Gynecologic Endocrinology 2018;5:26.

56. Wang W, Liao S, Sun W. Study on the prevalence and clinical characteristics of polycystic ovary syndrome in infertile women. Compilation of papers of the 11th National Conference on Endocrinology of The Chinese Medical Association; 2012.

57. Li B. Etiology screening and analysis of female infertility. Guide of China Medicine 2013;11:597-8.

58. Jiang A, Reng C, Liu F, et al. Analysis of Etiological Factor of Female Primary Infertile. Acta Academiae Medicinae Weifang 2007;29:59-60.

59. Zhao R, Wu Y, Mo W, et al. Analysis of etiological factor for 439 cases of female primary infertility. China Practical Medical 2008;3:52-3.

60. Teng X, Zhang P, Liu W, et al. An epidemiological study on polycystic ovary syndrome among nurses of 
childbearing age in Yan Chai Hospital. Proceedings of the 16th National Academic Conference of Chinese Diabetes Association; 2012.

61. Li X, Liang X, He Y. Investigation on prevalence and clinical characteristics of women at childbearing age with polycystic ovary syndrome in Ronggui area. Maternal \& Child Health Care of China 2012;27:3776-8.

62. Hao C. Correlation study on PCOS incidence and clinical characteristics of Han women of childbearing age in Shandong province. Shandong: Shandong University; 2006.

63. Li W, Hu H, Ma Z, et al. An epidemiological study on polycystic ovary syndrome in women of childbearing age in Shaoguan area. Heilongjiang Medicine 2016:646-8.

64. Wang L, Wei W, Wang G, et al. Prevalence of polycystic ovary syndrome and clinical characters of high estradiol level in general Han ethnic women in Shenzhen city. Qingdao Medical Journal 2015;47:417-9.

65. Ma L, Xiao Y, Meng P, et al. The analysis of disease pattern of gynecological outpatients at a hospital in Shenyang city. Chinese Journal of Health Management 2007;1:39-41.

66. Wang B. Epidemiological investigation and TCM syndrome analysis of Infertility in Suzhou area. Nanjing: Nanjing University of Traditional Chinese Medicine; 2019.

67. Jiang $X$. Investigation on the incidence of polycystic ovary syndrome among female middle school students in Suzhou city and analysis of related factors. Suzhou: The First Affiliated Hospital of Suzhou University; 2006.

68. Chen H. An epidemiological study on clinical characteristics and related factors of polycystic ovary syndrome in women of childbearing age in Tianjin. Tianjin: Tianjin Medical University; 2009.

69. Lin Y, Ni R, Yang X. Investigation on the incidence of polycystic ovary syndrome in women of childbearing age in Xinhe District. Chinese Community Doctors 2011;13:317.

70. Lin L, Mi N, Ding Y. Clinical characteristics of Han and Uygur child-bearing patients with polycystic ovary syndrome in Xinjiang. Journla of Chinese Practical Diagnosis and Therapy 2010;24:1154-7.

71. Guo Y, Gong A, Li R. Clinical analysis of related factors of polycystic ovary syndrome in Yi'nan county and its adjacent counties. Journal of Shandong Medical College 2016;38:66-9.

72. Wang G, Shi N. Investigation and analysis of adolescent polycystic ovary syndrome in Yinchuan city. China Health Care \& Nutrition 2016;26:56-7.
73. Feng G, Wang J, Zhai H. A Survey on Factors Affecting Future Fertility of Female College Students. Health Medicine Research and Practice 2016;13:32-4.

74. Zhang $\mathrm{P}$, Teng X, Wang H, et al. The prevalence of polyocystic ovary syndrome in nurses of reproductive age. Chinese Journal of Endocrinology and Metabolism 2013;29:206-10.

75. Song F, Chen Y, Li S, et al. A clinical study on the screening of polycystic ovary syndrome (PCOS) among college students in Hainan province by BMI and circumference method. World Latest Medicine Information 2019;54:72-3.

76. Wang L. Investigation and preliminary analysis of the related factors of polycystic ovary syndrome in women of childbearing age in Changsha city. Changsha: Central South University, 2012.

77. Zhang Y. A survey on the prevalence rate and related factors of PCOS in 5000 adolescent females in Changsha. Changsha: Hunan University of Chinese Medicine, 2009.

78. Wang Y, Zhao J, Yang W, et al. Epidemiological investigation and analysis on the sterility in flight attendants. Journal Of Civil Aviation Medicine 2016;26:247-9.

79. Jiao J, Fang Y, Wang T, et al. Epidemiologic investigation of polycystic ovarian syndrome (PCOS) in Han ethnic women of reproductive age in Liaoning Province, China. Clin Exp Obstet Gynecol 2014;41:304-9.

80. Zhuang J, Liu Y, Xu L, et al. Prevalence of the polycystic ovary syndrome in female residents of Chengdu, China. Gynecologic and obstetric investigation 2014;77:217-23.

81. Chen X, Yang D, Mo Y, et al. Prevalence of polycystic ovary syndrome in unselected women from southern China. Eur J Obstet Gynecol Reprod Biol 2008;139:59-64.

82. Sirmans SM, Pate KA. Epidemiology, diagnosis, and management of polycystic ovary syndrome. Clin Epidemiol 2013;6:1-13.

83. Rotterdam ESHRE/ASRM-Sponsored PCOS consensus workshop group. Revised 2003 consensus on diagnostic criteria and long-term health risks related to polycystic ovary syndrome (PCOS). Hum Reprod 2004;19:41-7.

84. Patel S. Polycystic ovary syndrome (PCOS), an inflammatory, systemic, lifestyle endocrinopathy. J Steroid Biochem Mol Biol 2018;182:27-36.

85. Ding T, Hardiman PJ, Petersen I, et al. The prevalence of polycystic ovary syndrome in reproductive-aged women of different ethnicity: a systematic review and meta-analysis. Oncotarget 2017;8:96351-8.

86. Nelson JW, Scammell MK, Hatch EE, et al. Social 
disparities in exposures to bisphenol A and polyfluoroalkyl chemicals: a cross-sectional study within NHANES 20032006. Environ Health 2012;11:10.

87. Yang Q, Zhao Y, Qiu X, et al. Association of serum levels of typical organic pollutants with polycystic ovary syndrome (PCOS): a case-control study. Hum Reprod 2015;30:1964-73.

88. Fernández M, Bourguignon N, Lux-Lantos V, et al. Neonatal exposure to bisphenol a and reproductive and endocrine alterations resembling the polycystic ovarian syndrome in adult rats. Environ Health Perspect 2010;118:1217-22.

89. Kirby JB, Kaneda T. Neighborhood socioeconomic disadvantage and access to health care. J Health Soc Behav 2005;46:15-31.

90. Legro RS, Kunselman AR, Dodson WC, et al. Prevalence and predictors of risk for type 2 diabetes mellitus and

Cite this article as: $\mathrm{Wu} \mathrm{Q}$, Gao J, Bai D, Yang Z, Liao Q. The prevalence of polycystic ovarian syndrome in Chinese women: a meta-analysis. Ann Palliat Med 2021;10(1):74-87. doi: 10.21037/apm-20-1893 impaired glucose tolerance in polycystic ovary syndrome: a prospective, controlled study in 254 affected women. J Clin Endocrinol Metab 1999;84:165-9.

91. Ma L, Meng P, Duan Y, et al. Study on the related factors of polycystic ovary syndrome. Medicine of Shenyang Army 2009;22:64-6.

92. Goodarzi MO, Dumesic DA, Chazenbalk G, et al. Polycystic ovary syndrome: etiology, pathogenesis and diagnosis. Nat Rev Endocrinol 2011;7:219-31.

93. Lin Y, Li L, Xu R. The Relationship between Changes in Sex Hormone Level and Physiology and Disease of Women in Different Age Groups. Henan Journal of Preventive Medicine 2010;21:179-81.

94. Bani Mohammad M, Majdi Seghinsara A. Polycystic ovary syndrome (PCOS), diagnostic criteria, and AMH. Asian Pac J Cancer Prev 2017;18:17-21. 